\title{
Dosimetric comparison of fixed field dynamic IMRT and VMAT techniques in simultaneous integrated boost radiotherapy of Prostate Cancer
}

Haitao SUN ( $\sim 1536678860 @ q q . c o m$ )

Zhongshan Hosipital of Traditional Chinese Medicine

Ying Liu

the fifth affiliated hospital of Guangzhou Medical University

Along Chen

Sun Yat-sen University Cancer Center

Ning Wang

Shaoxing City Keqiao District Hospital of Traditional Chinese Medicine

\section{Research}

Keywords: Intensity modulated radiotherapy; Volumetric modulated arc therapy; Prostate cancer;

Simultaneously integrated boost.

Posted Date: May 6th, 2020

DOI: https://doi.org/10.21203/rs.3.rs-25930/v1

License: (c) (i) This work is licensed under a Creative Commons Attribution 4.0 International License.

Read Full License 


\section{Abstract}

Background: As the high risk prostate cancer can be benefited from the combination of hypofractionated radiotherapy and pelvic conventional fraction radiotherapy, the comparison between fixed field dynamic IMRT and VMAT techniques can provide suggestion for clinical treatment.

Methods: We selected 10 patients with high risk prostate cancer who received radiotherapy at Sun Yatsen University Cancer Center from 2013 January to 2013 December. The target including the prostate, seminal vesicle and pelvic lymph nodes. With the same prescription and optimized parameters, 9 field IMRT, single arc and double arc VMAT treatment plans were designed, which are expressed by 9F, 1ARC and 2ARC respectively. The dose distribution of the targets, organs at risk (OAR), monitor units (MUs), treatment time and gamma pass ratios of dose verification were compared.

Results: The $\mathrm{D}_{2 \%}(69.37 \pm 0.89) \mathrm{Gy}, \mathrm{D}_{50 \%}(66.92 \pm 0.63) \mathrm{Gy}, \mathrm{HI}(0.09 \pm 0.02)$ and $\mathrm{Cl}(0.83 \pm 0.05)$ of $\mathrm{PTV} 1$ in $9 \mathrm{~F}$ were slightly better than those of 1ARC which were (71.13 \pm 1.21$) \mathrm{Gy},(68.50 \pm 0.76) \mathrm{Gy},(0.12 \pm 0.02)$, $(0.74 \pm 0.07)$, except $D_{98 \%}$, the difference were significant $(p<0.05)$. All dosimetric indices of PTV1 in $9 F$ and 2ARC were close and has no significant differences $(p>0.05)$. The $V_{95}(99.45 \pm 0.78) \%$ of PTV2 in $9 F$ was slightly better than that in 1 ARC $(99.35 \pm 1.28) \%$, the difference was significant $(p<0.05)$. All dosimetric indices of PTV2in 9F and 2ARC were close and the difference were no significant $(p>0.05)$. The $D_{\text {mean }}$ of bladder and the $V_{67.5 G y}$ of rectum between all three plans were similarity The $D_{\text {mean }}$ of left and right femoral in $1 A R C$ and $2 A R C$ were lower than that in $9 F$, and the difference was significant $(p<0.05)$. Other dosimetric indices of OARs in 9F were lower than those in $1 A R C$ and $2 A R C$, and much lower than 1ARC, the difference were significant $(p<0.05)$. The mean monitor units in 1ARC and 2ARC were fewer by $70.0 \%$ and $67.2 \%$ in comparison with $9 \mathrm{~F}$. The treatment mean time in $1 \mathrm{ARC}$ and $2 \mathrm{ARC}$ were shorter by $81.7 \%$ and $61 \%$ in comparison with $9 \mathrm{~F}$. The verification pass ratios of $\mathrm{\gamma}(3 \% / 3 \mathrm{~mm})$ were $97.8 \%$ (9F), $98.9 \%(1 A R C)$ and $99.4 \%(2 A R C)$ respectively, the difference were significant $(p<0.05)$.

Conclusion: Compared with IMRT, VMAT improved delivery efficiency noticeably. Two arcs provided comparable tumor dosimetric coverage, but performed worse in dose sparing for bladder, rectum and small bowel. IMRT plan was better than VMAT in prostate cancer simultaneous integrated boost radiotherapy.

\section{Background}

Prostate cancer is the most common noncutaneous cancer in men, with 1,212,653 case excepted in 2019 in China alone. Treatment of localized prostate cancer has been proven by clinical trial including hypofractionation RT dose escalation with ENI and ADT combined with RT[1-5]. With the development of radiotherapy IMRT, it has been generally used in prostate cancer. Radiation therapy related toxicities is associated with high total RT dose, short recovery time, and the volume of neighboring OAR normal tissues (rectum, bowel and bladder) ever in prostate-only $\mathrm{RT}^{6,7}$.In recent years, with the development of radiotherapy technology, IMRT has replaced 3-dimensional conformal radiation therapy as the most 
common method of radiation therapy for prostate cancer for its conformable dose distributions which can reduce normal tissue toxicity[1, 3]. The most common method for IMRT delivery for prostate cancer involves [5-9], fixed gantry positions with computer-generated, sliding-window multi-leaf collimator positions to modulate dose to the prostate [8-10]. A more recent IMRT technique named as VMAT, involves gantry rotation around the prostate with 1 to 4 arcs while the $x$-ray beam is on. In VMAT technique the dose rate varies while the gantry moving around. Palma et al reported that the most favorable equivalent uniform doses and lowest doses to organs at risk were achieved with variable dose rate VMAT, which was statistically significantly better than 5-field, IMRT for rectal and femoral head endpoints and better than constant dose-rate VMAT for most bladder and rectal endpoints [1113].Multiple groups have observed that VMAT reduces beam-on time and the radiation dose relative to 7 to 9 field IMRT.

According to NCCN guidelines, doses of 75.6 to $79.2 \mathrm{~Gy}$ in conventional fractions to the prostate are appropriate for patients with low-risk cancers. For patients with intermediate or high-risk disease, doses up to 81.0 Gy provide improved PSA-assessed disease control. For radical radiotherapy, patients with lowrisk cancer should not receive pelvic lymph node irradiation or ADT, patients with intermediate-risk cancer may be considered for pelvic lymph node irradiation and 4 to 6 month neoadjuvant/concomitant/adjuvant ADT. Patients with high-risk cancers are candidates for pelvic lymph node irradiation and the addition of neoadjuvant /concomitant/adjuvant ADT for a total of 2-3y [14].The target area of this study include prostate, seminal vesicle and pelvic lymph nodes. The studies were irradiated simultaneously, the former group is treated with conventional segmented irradiation of pelvic cavity and prostate, and then with local prostate boost, the latter group is also treated with conventional segmented irradiation. In this way, patients are treated longer and more expensive. We have to make two plans for one patient, which increase the workload of doctors and physicists. At present, several published studies have been shown that the effect of increasing single dose or reducing the total dose is similar to that of conventional fractionated irradiation and the toxicity is acceptable;Meanwhile, the second edition of NCCN guidelines for prostate cancer in 2014 also included the content of hypo-fraction radiotherapy for prostate cancer (2.4-4Gy / fraction, treatment period of 4-6 weeks). Hypo-fraction can not only shorten the treatment time and reduce the medical cost, but also improve the efficiency of medical resources. The combination of conventional segmented irradiation of pelvic cavity and the hypofraction radiotherapy of prostate will be more beneficial for the patients. In this paper, we compared the difference of dosimetry and treatment efficiency of the IMRT and VMAT technology which designed through Varian treatment plan system.

\section{Methods}

Patients selection and CT simulation

We selected 10 patients with high risk prostate cancer who received radiotherapy at Sun Yat-sen University Cancer Center from 2017-01-04, aged $62 \sim 78$ years, median age 70 years. All of those patients didn't go through radical prostatectomy. Planning CT scans were performed at $5 \mathrm{~mm}$ slice thickness 
using a dedicated helical CT scanner, from the upper abdomen to $5 \mathrm{~cm}$ below the Ischia tuberosities after immobilization with Knee and feet support immobilization devices. CT images were transferred to the inverse TPS through network. Patients were instructed to have a comfortably full bladder and an empty rectum at $\mathrm{CT}$ acquisition and before each treatment. The bladder was filled to make the small intestine move downwards to reduce the volume of small intestine irradiation.

\section{Contouring and Planning}

The CTV, CTV1 was defined as the entire prostate and seminal vesicle (if involved); CTV2 included the whole prostate and seminal vesicle (if not involved). PTV1 was generated by adding anisotropic $0.5 \mathrm{~cm}$ margin to the CTV1 apart from prosteriorly where $0.3 \mathrm{~cm}$ margin was added (to decrease prostate-rectal interface dose). PTV2 was a $0.7 \mathrm{~cm}$ anisotropic expansion from CTV2 except posteriorly $(0.5-0.7 \mathrm{~cm})$ depending on rectal fullness. The prescribed dose of PTV1 was 67.5Gyin 25 daily fraction and PTV2 was $47.5 \mathrm{~Gy}$ in 25 daily fraction. Contouring of the OAR followed the RTOG pelvic normal tissue contouring guidelines [15]. The rectum was outlined from the level of the ischial tuberosities to the rectosigmoid flexure. The whole bladder was contoured; femoral heads were delineated to the level of the ischial tuberosities. The bowel was contoured as the entire volume of peritoneal space to within $1 \mathrm{~cm}$ of the cranial margin of the nodal PTV. The TPS is Varian Eclipse (version 10.1); The radiotherapy equipment is Varian Trilogy linear accelerator with 120 Multi-leaf Collimator, and the isocenter accuracy is $5 \mathrm{~mm}$. Three intensity modulated treatment plans were designed for each case using 6MV X-ray, that were fixed field dynamic intensity modulation 9-field plan (9F), VMAT single-arc plan (1ARC) and double-arc plan (2ARC), respectively,. The maximum dose rate was set at $600 \mathrm{MU} / \mathrm{min}$. Among them, for IMRT, 9F beams were treated using a dynamic multi-leaf collimator and the radiation directions was $160^{\circ}, 120^{\circ}, 80^{\circ}, 40^{\circ}, 0^{\circ}$, $320^{\circ}, 280^{\circ}, 240^{\circ}, 200^{\circ}$ respectively; For VMAT, the 1 ARC adopts a single arc of $179^{\circ}$ to $181^{\circ}$ anticlockwise rotation and for the 2ARC, the first arc rotated from $179^{\circ}$ anticlockwise to $181^{\circ}$, than the second arc rotated clockwise from $181^{\circ}$ to $179^{\circ}$. During the rotation of gantry, the step length of the subfield segment is $2^{\circ}$. There were all adopt the simultaneous integrated boost technology. The minimum allowable dose in the PTV was $93 \%$ of the prescribed dose and the maximum allowable dose in the PTV was $115 \%$ of the prescribed dose. At least $95 \%$ of the PTV received $>95 \%$ of the prescribed dose. For OAR, bladder $\mathrm{V}_{55} \mathrm{~Gy}<$ $30 \%, V_{67.5}$ Gy $<10 \%$;Rectum $V_{55} G y<30 \%, V_{67.5} G y<10 \%$;Left and right femoral head $V_{40}$ Gy $<5 \%$; The small intestine $D_{\max }<50 \mathrm{~Gy}$. The dose constraint and optimization parameters of the three plans were the same, and the dose calculation grid size was $2.5 \mathrm{~mm}$, using the AAA dose algorithm ${ }^{12}$.

Plan quality evaluation

For the sake of convenience, the three plans were normalized after the completion, at least $95 \%$ of the PTV1 received $>95 \%$ of the prescribed dose. According to the ICRU83 report [16], the dose distribution in the target area was evaluated with the maximum dose D2\%, the minimum dose D98\% and the median dose $\mathrm{D}_{50} \%, \mathrm{Cl}$ and $\mathrm{HI}$ are introduced to evaluate the planned dose distribution, where $\mathrm{HI}=\left(\mathrm{D}_{2} \%-\mathrm{D}_{98} \%\right) /$ $\mathrm{D} 50 \%, \mathrm{Cl}=\left(\mathrm{TV}_{\mathrm{Rl}} \times \mathrm{TV}_{\mathrm{Rl}}\right) /\left(\mathrm{TV} \times \mathrm{V}_{\mathrm{Rl}}\right)\left(\mathrm{D}_{\mathrm{X}} \%\right.$ is the dose received by $\mathrm{X} \%$ of the target volume, $\mathrm{TV}_{\mathrm{RI}}$ represents the target volume within the prescription isodose volume, TV is the volume of the target area PTV, and $\mathrm{V}_{\mathrm{RI}}$ 
is the volume enclosed by $95 \%$ of the prescription dose line). The smaller the $\mathrm{HI}$ value is, the better the uniformity of the dose distribution in the target area is. The value of $\mathrm{Cl}$ is between 0 and 1 , which represents the ideal situation that the target volume coincides exactly with the treatment volume. If $\mathrm{Cl}$ equals zero that represents a plan in which there is no overlap between the two volumes. Dose constraints were also evaluated for each plan, $\mathrm{V}_{50} \mathrm{~Gy}, \mathrm{~V}_{55} \mathrm{~Gy}, \mathrm{~V}_{60} \mathrm{~Gy}, \mathrm{~V}_{67.5} \mathrm{~Gy}$ and $\mathrm{D}_{\text {mean }}$ were used to evaluate rectum and bladder, $V_{40}$ Gy and $D_{\text {mean }}$ were used to evaluate left and right femoral head, and $D_{\text {max }}$ and $D_{\text {mean }}$ were used to evaluate small intestine. $D_{\text {mean }}$ was the average dose received by $O A R$, and $D_{\max }$ was the maximum point dose received by OAR. Record the number of subfields, MU and effective treatment time (the time from the beginning of beam out to the end of beam out after the completion of patient positioning), and compare the results of each execution parameter of the three groups. The semiconductor three-dimensional detector array dose verification system ArcCheck was used to conduct dose verification for all plans. The maximum dose point was taken as the standard, and the dose threshold was set to $10 \%$. The DTA and Gamma pass rates of each plan were compared under the standard of $3 \% / 3 \mathrm{~mm}$.

Statistical method

Spss19.0 software was used for statistical analysis. Shapiro-Wilk test was used to test the difference of paired data of each index of treatment plan (9F and $1 A R C, 9 F$ and $2 A R C$ ). If it is in line with the normal distribution, $T$ test was used to test the results of $9 F$ and $1 A R C, 9 F$ and $2 A R C$, and Wilcoxon rank sum test was used to test the results of non normal distribution $(P<0.05)$.The difference was statistically significant, and the data results were expressed as mean \pm standard deviation $(\bar{x} \pm s)$.

\section{Result}

Dosimetric comparison of target area

The mean volume of PTV (including PTV1 and PTV2) was $1153.2 \pm 146.3 \mathrm{~cm}^{3}\left(869.7 \sim 1409.0 \mathrm{~cm}^{3}\right)$, which was large and complex. Figure 1 shows the isodose distributions on axial images of three plans. PTV1 shown as red line, PTV2 shown as green line.PTV1 was inside PTV2. The isodose lines were displayed on an absolute dose scale, the isodose levels increased by $20 \mathrm{~Gy} \sim 70.88 \mathrm{~Gy}$ gradient were shown. As the dosimetric comparison of 9F with 1ARC and 2ARC is shown in Table 1: For PTV1, 9F plans were similar to 2ARC plans, there was no statistical significance $(P>0.05)$. And the $D_{98 \%}$ of the three plans indices did not show any obvious difference. The $D_{2}$ and $D_{50 \%}$ of $9 F$ and $2 A R C$ were closer to the prescription dose, and the $\mathrm{HI}$ and $\mathrm{Cl}$ were slightly better than $1 \mathrm{ARC}$, and the differences were statistically significant $(P<0.05)$. For $P T V 2, V_{95 \%}$ of $9 F$ was better than $1 A R C$, the difference was statistically significant $(P<0.05)$, but $D_{98 \%}$ has no statistical significance $(P>0.05)$, 9F and $2 A R C$ has no statistical significance $(P>0.05)$.

Dosimetric comparison of OAR 
The mean results of OARs for dosimetric comparison of 9F with 1ARC and 2ARC plans are listed in Table 2. The three kinds of plans all met the clinical needs. For rectum $\mathrm{V}_{67.56 \mathrm{y}}, 9 \mathrm{~F}$ was lower than $2 \mathrm{arc}$, but the difference was not statistically significant $(P>0.05)$; for bladder $D_{\text {mean }}$, there was no statistically significant difference between the three groups $(P>0.05)$; for left and right femoral head $D_{\text {mean }}, 1 A R C$ and 2ARC were lower than $9 F$, the difference was statistically significant $(P<0.05)$. Other OAR parameters evaluation,the $9 \mathrm{~F}$ plans were lower than $1 \mathrm{ARC}$ and $2 \mathrm{ARC}$, and the difference was statistically significant compared with 1ARC $(P<0.05)$.

Comparison of radiotherapy efficiency and dose

Compared with 9F, 1 ARC and 2ARC the monitor units (MUs) was reduced $70.0 \%$ and $67.2 \%$, accounting for $9 \%$ and $18 \%$ of the number of subfields respectively. The average delivery time of the $9 F, 1 A R C$, and 2ARC plans was $449.3 \pm 29$ seconds, $82.2 \pm 0.8$ seconds, and $175.0 \pm 0.9$ seconds, respectively, reduced by $81.7 \%$ and $61 \%$, relative to the $9 F$. The gamma and DTA pass rate for these plans exhibited were measured using the ArcCheck detector along with ion chamber measurements. The average pass rate was greater than $95 \%$ under the standard of $3 \% / 3 \mathrm{~mm}$, and 1ARC and 2ARC were slightly higher than 9F.The differences of the above evaluation indicators were statistically significant $(p<0.05)$, as shown in Table 3.

Table 1

Dosimetric comparison of $9 \mathrm{~F}$ with $1 \mathrm{ARC}$ and $2 \mathrm{ARC}$ target areas $(\bar{x} \pm \mathrm{s})$

\begin{tabular}{|llllllll|}
\hline Parameter & $9 F$ & 1ARC & t/z & P & 2ARC & t/z & P \\
\hline PTV1 & & & & & & & \\
\hline$D_{2 \%} / G y$ & $69.37 \pm 0.89$ & $71.13 \pm 1.21$ & -4.865 & 0.001 & $69.15 \pm 0.79$ & -0.561 & $0.575^{a}$ \\
\hline$D_{98 \%} / G y$ & $63.18 \pm 0.55$ & $63.03 \pm 0.31$ & 0.86 & 0.412 & $63.31 \pm 0.20$ & -0.965 & 0.360 \\
\hline$D_{50 \%} / G y$ & $66.92 \pm 0.63$ & $68.50 \pm 0.76$ & -6.598 & 0.000 & $67.20 \pm 0.55$ & -1.730 & 0.118 \\
\hline HI & $0.09 \pm 0.02$ & $0.12 \pm 0.02$ & -3.406 & 0.008 & $0.09 \pm 0.02$ & 1.406 & 0.193 \\
\hline Cl & $0.83 \pm 0.05$ & $0.74 \pm 0.07$ & 3.866 & 0.004 & $0.79 \pm 0.08$ & 1.850 & 0.097 \\
\hline PTV2 & & & & & & & \\
\hline$V_{95 \%} / \%$ & $99.45 \pm 0.78$ & $99.35 \pm 1.28$ & 2.549 & 0.031 & $99.33 \pm 0.75$ & 0.448 & $0.888^{a}$ \\
\hline$D_{98 \%} / G y$ & $46.13 \pm 0.65$ & $45.70 \pm 1.24$ & 0.959 & 0.363 & $46.02 \pm 6.14$ & 0.442 & 0.669 \\
\hline Note: a is non-normal distribution data, and $\mathrm{z}$ is non-parametric test statistics & & \\
\hline
\end{tabular}


Table 2

dosimetric comparison of 9F with 1ARC and 2ARC plans for organs at risk $\overline{(x} \pm s)$

\begin{tabular}{|c|c|c|c|c|c|c|c|}
\hline Parameter & $9 \mathrm{~F}$ & 1ARC & $t / z$ & $\mathbf{p}$ & 2ARC & $t / z$ & $\mathbf{p}$ \\
\hline \multicolumn{8}{|l|}{ Rectum } \\
\hline $\mathrm{V}_{50 \mathrm{~Gy}} / \%$ & $19.78 \pm 3.55$ & $28.2 \pm 1.45$ & -8.949 & 0.000 & $25.83 \pm 0.81$ & -2.983 & $<0.001$ \\
\hline $\mathrm{V}_{55 \mathrm{~Gy}} / \%$ & $15.02 \pm 3.14$ & $21.19 \pm 1.88$ & -10.851 & 0.000 & $19.90 \pm 1.41$ & -5.885 & $<0.001$ \\
\hline $\mathrm{V}_{60 \mathrm{~Gy}} / \%$ & $10.48 \pm 2.39$ & $15.08 \pm 2.36$ & -7.606 & 0.000 & $14.07 \pm 1.83$ & -6.771 & $<0.001$ \\
\hline $\mathrm{V}_{67.5 \mathrm{~Gy}} / \%$ & $0.99 \pm 1.21$ & $3.91 \pm 2.40$ & -4.324 & 0.002 & $1.72 \pm 1.90$ & -1.262 & $0.207^{a}$ \\
\hline $\mathrm{D}_{\text {mean }} / \%$ & $44.63 \pm 1.00$ & $47.21 \pm 2.21$ & -4.339 & 0.002 & $45.96 \pm 1.45$ & -3.708 & 0.005 \\
\hline \multicolumn{8}{|l|}{ Bladder } \\
\hline $\mathrm{V}_{50 \mathrm{~Gy}} / \%$ & $28.97 \pm 5.21$ & $45.11 \pm 12.55$ & -2.803 & $0.005^{a}$ & $37.54 \pm 4.96$ & -4.011 & 0.003 \\
\hline$V_{55 G y} / \%$ & $18.56 \pm 2.64$ & $24.73 \pm 1.85$ & -6.923 & 0.000 & $20.81 \pm 3.06$ & -3.011 & 0.015 \\
\hline $\mathrm{V}_{60 \mathrm{~Gy}} / \%$ & $13.66 \pm 2.97$ & $16.64 \pm 3.34$ & -5.415 & 0.000 & $14.46 \pm 2.76$ & -3.863 & 0.004 \\
\hline $\mathrm{V}_{67.5 \mathrm{~Gy}} / \%$ & $0.88 \pm 0.85$ & $6.25 \pm 2.53$ & -5.619 & 0.000 & $3.09 \pm 1.28$ & -3.946 & 0.003 \\
\hline $\mathrm{D}_{\text {mean }} / \mathrm{Gy}$ & $48.81 \pm 2.00$ & $47.99 \pm 3.72$ & 0.908 & 0.387 & $47.55 \pm 3.00$ & 1.932 & 0.085 \\
\hline \multicolumn{8}{|c|}{ LeftFemoral Head } \\
\hline $\mathrm{V}_{40 \mathrm{~Gy}} / \%$ & $1.8 \pm 0.74$ & $3.39 \pm 1.01$ & -4.235 & 0.002 & $2.49 \pm 0.74$ & -2.697 & 0.024 \\
\hline $\mathrm{D}_{\text {mean }} / \mathrm{Gy}$ & $26.43 \pm 3.54$ & $23.68 \pm 3.94$ & 2.814 & 0.020 & $23.22 \pm 2.81$ & 3.980 & 0.003 \\
\hline \multicolumn{8}{|c|}{ Right Femoral Head } \\
\hline $\mathrm{V}_{40 \mathrm{~Gy}} / \%$ & $1.75 \pm 0.74$ & $3.37 \pm 0.86$ & -4.313 & 0.002 & $2.44 \pm 0.89$ & -2.531 & 0.032 \\
\hline $\mathrm{D}_{\text {mean }} / \mathrm{Gy}$ & $26.34 \pm 3.27$ & $22.05 \pm 3.54$ & 5.449 & 0.000 & $22.73 \pm 3.58$ & 4.739 & 0.001 \\
\hline \multicolumn{8}{|c|}{ Small Intestine } \\
\hline $\mathrm{D}_{\max } / \mathrm{Gy}$ & $44.05 \pm 7.63$ & $45.99 \pm 9.07$ & -2.983 & 0.018 & $45.18 \pm 7.79$ & -2.379 & 0.045 \\
\hline
\end{tabular}


Table 3

comparison of $9 \mathrm{~F}$ with $1 \mathrm{ARC}$ and $2 \mathrm{ARC}$ treatment efficiency and dose validation $\overline{(x} \pm \mathrm{s})$

\begin{tabular}{|c|c|c|c|c|c|c|c|}
\hline Parameter & $9 \mathrm{~F}$ & 1ARC & $t / z$ & $p$ & 2ARC & $t / z$ & $p$ \\
\hline Monitor Units & $\begin{array}{l}1794.8 \pm \\
155.2\end{array}$ & $\begin{array}{l}534.5 \pm \\
76.5\end{array}$ & 37.009 & $<.001$ & $\begin{array}{l}588.3 \pm \\
155.0\end{array}$ & 22.308 & $<.001$ \\
\hline $\begin{array}{l}\text { Segment } \\
\text { Number }\end{array}$ & $\begin{array}{l}1907.5 \pm \\
94.8\end{array}$ & $\begin{array}{l}178.0 \pm \\
0.0\end{array}$ & 57.685 & $\begin{array}{l}<.001 \\
0.00\end{array}$ & $356.0 \pm 0.0$ & 51.748 & $\begin{array}{l}<.001 \\
0.01\end{array}$ \\
\hline $\begin{array}{l}\text { Treatment time } \\
\text { /s }\end{array}$ & $449.3 \pm 29.0$ & $82.2 \pm 0.8$ & 31.290 & $\begin{array}{l}< \\
0.001\end{array}$ & $175.0 \pm 0.9$ & 22.747 & $<.001$ \\
\hline $\mathrm{DTA}(3 \% / 3 \mathrm{~mm})$ & $96.2 \pm 1.2$ & $97.9 \pm 0.5$ & -2.201 & $0.028^{a}$ & $99.1 \pm 0.4$ & -2.201 & $0.028^{\mathrm{a}}$ \\
\hline$\gamma(3 \% / 3 \mathrm{~mm})$ & $97.8 \pm 0.6$ & $98.9 \pm 0.4$ & -5.329 & 0.003 & $99.5 \pm 0.3$ & -9.463 & $<.001$ \\
\hline
\end{tabular}

\section{Discussion}

With the volumetric modulated arc therapy widely used in clinical, there are many literatures at home and abroad comparing its difference with the intensity modulated radiation therapy, including dosimetry, treatment efficiency and dose validation pass rate. A number of previous studies have shown that in head and neck tumors and esophageal cancer, the dose distribution of VMAT is equal to or slightly better than that of IMRT, which can greatly shorten the treatment time and improve the treatment efficiency. But, the previously reported target structure is often relatively simple. The research of Guckenberger and Bortfeld shows that VMAT may not provide enough intensity modulation for more complex targets $[13-14,19-$ 21]. In order to obtain a shorter treatment time, single arc VMAT may over sacrifice the quality of dose distribution; increasing the number of subfields or rotating arcs can improve the quality of dose distribution, However, the treatment time will increase correspondingly .

In the comparative study of radiotherapy techniques for prostate cancer, different conclusions were drawn according to the target areas of different structures and shapes. Studies only including prostate or prostate and seminal vesicle, Bedford et al [13] have shown that single-arc VMAT had better PTV coverage and less OAR exposure dose than 5-field IMRT; Boylan et al [10] have shown that single arc VMAT can better protect OAR compared with 5-field IMRT, but the PTV coverage was worse; other reports had shown that VMAT and IMRT have similar dose distribution, and with the number of fixed fields increases, the dose distribution of IMRT will gradually be equal to or even better than VMAT. Yoo et al [17] used sequential irradiation to compare the dose distribution and treatment efficiency of IMRT with single arc and double arc VMAT. The primary planning target volume contained prostate, seminal vesicles, and pelvic lymph node with a margin. The results showed that IMRT could protect bladder, rectum and small intestine, and had similar $\mathrm{HI}$ and $\mathrm{Cl}$ to 2ARC, slightly better than 1ARC. For the second course plan, 2ARC 
and IMRT had similar dose distribution. The results of our study aresimilar to Yoo et al: the target area coverage of IMRT is no less than or slightly better than 1ARC, similar to 2ARC; except for $D_{\text {mean }}$ of left and right femoral head and $D_{\text {mean }}$ of bladder, other parameters of OAR are better than $1 A R C$ and $2 A R C$, and significantly better than $1 A R C$, which is better protection of the bladder, rectum and small intestine. In addition, those plans were normalized such that the prescription dose covered at least $95 \%$ of the PTV, after which one plan with D5 of PTV $\leq 110 \%$ and better OAR sparing was selected for each technique. As shown in Fig. 1, IMRT will be exposed to relatively more low fluenceregion of $20 \mathrm{~Gy}$ and $30 \mathrm{~Gy}$, and the smoothness of dose curve will be worse. Compared with IMRT, double arc VMAT can significantly reduce the radiation dose of OAR, which is different from this result, the reason may be that the IMRT used in this study is $5 \mathrm{~F}$ and the number of subfields is less. In general, the more field shots, the better the result.

The biggest advantage of VMAT is to greatly shorten the treatment time. This study shows that compared with IMRT, the average treatment timeof 1 ARC and 2ARCshorten by $81.7 \%$ and $61 \%$, and also reduce the number of MUs by $70.0 \%$ and $67.2 \%$ respectively, which can reduce the loss of the treatment machine. Those results are similar to the results reported by Yoo et al [17], but Quan et al [9] used AIP algorithm on Pinnacle v9.0 system to compare with VMAT and IMRT, VMAT had $30 \%$ more MUs than 8 field IMRT, but the treatment time was reduced 3 minutes. In addition, the three-dimensional dose validation results of three groups plans in this study meet the clinical requirements ( $Y \geq 90 \%)$. Although VMAT plan is more complex than IMRT plan and involves more parameters (collimator angle, multi-leaf grating, dose rate, gantry rotation speed) in the process of treatment implementation, the measurement results of VMAT plan are better than IMRT, which may be because Arccheck is cylindrical for the phantom, the subfield in VMAT is smaller, and the angle difference of probe dose response is smaller, which makes it more suitable for the measurement of VMAT plan [23-24].

In conclusion, under the condition that the prescription dose covered at least $95 \%$ of the PTV standard, whether using IMRT or VMAT technology in simultaneous integrated boost radiotherapy for prostate cancer can satisfy the needs of OAR and have a good dose verification pass rate. Compared with the IMRT plan, VMAT plan can reduce the treatment time significantly and improve the treatment effectively; 2ARC plan has similar target coverage; but the protection of bladder, rectum and small bowel is worse; the less the number of VMAT arcs, the worse the OAR protection, With the number of arcs increases the quality of plan improves, but at the same time, it the number of MUs and treatment time also increases. The results of this study show that for the complex target structure including prostate, seminal vesicle and pelvic lymph node drainage area, the use of IMRT technology can significantly improve the quality of planning, and can better protect the OAR, and is more suitable for the simultaneous integrated boost radiotherapy of prostate cancer pelvic radiation prevention. However, considering that the sample size used in this study is small the results need to be further verified by expanding the sample size.The plan designer needs to compare the advantages and disadvantages of VMAT and IMRT first with a larger sample size for cases with different target size or structure, weigh the gains and losses, and finally select a more appropriate treatment technology. 


\section{Conclusions}

Compared with IMRT, VMAT noticeably improved delivery efficiency, with two arcs provided comparable tumor dosimetric coverage, performed worse in dose sparing for bladder, rectum and small bowel. IMRT plan was better than VMAT in prostate cancer radiotherapy using a simultaneous integrated boost.

\section{Abbreviations}

RT: Radiation therapy; ENI: Elective Nodal Irradiation; ADT: Androgen deprivation therapy; CT: Computed tomography; PTV: Planning target volume; CTV: Clinical target volume; GTV: Gross tumor volume; VMAT: Volumetric modulated arc therapy; Gy: Gray; IMRT: Intensity modulated radiotherapy; TPS: treatment planning system; $\mathrm{HI}$ : Homogeneity index; $\mathrm{Cl}$ : conformability index; MUखmonitor unit囚OAR囚Organs at risk

\section{Declarations}

\section{Acknowledgements}

Not applicable.

\section{Funding}

No funding was received.

\section{Availability of data and materials}

The datasets used and analyzed during the current study are available from the corresponding author on reasonable request

\section{Authors' contributions}

SUN and WN analysed and interpreted the data, performed the statistical analysis and were major contributors in writing the manuscript. LY and CHEN helped with the statistical analysis and writing the manuscript. SUN, WN, LY, and CHEN helped drafting the manuscript. All authors read and approved the final manuscript.

\section{Ethics approval and consent to participate}

Not applicable

\section{Consent for publication}

Not applicable

Competing interests 
The authors declare that they have no competing interests.

\section{Publisher's Note}

Springer Nature remains neutral with regard to jurisdictional claims in published maps and institutional affiliations.

\section{Author details}

1.Radiotherapy Room, Department of Medical Imaging, Zhongshan Traditional Chinese Medicine Hospital, Zhongshan 528400, China2. The Department of the Radiotherapy $\otimes$ The Fifth Affiliated Hospital of Guangzhou Medical University, Guangzhou 510060, China3. State Key Laboratory of Oncology in South China, Department of Radiation Oncology, Cancer Center, Sun Yat-sen University, Guangzhou 510060, China

\section{References}

1. Mellon EA, Javedan $\mathrm{K}$, Strom TJ, et al. A dosimetric comparison of volumetric modulated arc therapy with step-and-shoot intensity modulated radiation therapy for prostate cancer[J]. Pract Radiat Oncol, 2015, 5(1):11-15.

2. Hegazy MW, Mahmood RI, Al Otaibi MF, et al. Hypofractionated Volumetric Modulated Arc Radiotherapy with simultaneous Elective Nodal Irradiation is feasible in prostate cancer patients: A single institution experience[J]. J Egypt Natl Canc Inst, 2016, 28(2):101-110.

3. Olivera J, Penedo JM, Marin JP, et al. Localized Prostate Cancer: Volumetric Modulated Arc Therapy (VMAT) Versus Intensity Modulated Radiation Therapy (IMRT)-Which One is Better[J]. Int J Radiat Oncol Biol Phys, 2014, 90(1):S863.

4. Gautam B. Literature review on IMRT and VMAT for prostate cancer[J]. American Journal of Cancer Review, 2014, 2(1): 1-5.

5. Fossati N, Karnes R J, Colicchia M, et al. Impact of Early Salvage Radiation Therapy in Patients with Persistently Elevated or Rising Prostate-specific Antigen After Radical Prostatectomy[J]. 2017.

6. Amaloo C, Nazareth DP, Kumaraswamy LK. Comparison of hybrid volumetric modulated arc therapy (VMAT) technique and double arc VMAT technique in the treatment of prostate cancer.[J]. Radiol Oncol, 2015, 49(3):291-8.

7. Lee S, Shim J, Chang K, et al. Which is an optimizing modality for IMRT prostate case in linac step and shoot, tomotherapy, RapidArc, VMAT, and proton using new plan evaluation scoring index and dosimetric physical index?[J]. Int J Radiat Oncol Biol Phys, 2014, 90(1):S874.

8. James C.L. Chow, Runqing Jiang. Dose-volume and radiobiological dependence on the calculation grid size in prostate VMAT planning[J]. Medical Dosimetry Official Journal of the American Association of Medical Dosimetrists, 2018, 43(4). 
9. Quan EM, Li X, Li Y, et al. A comprehensive comparison of IMRT and VMAT plan quality for prostate cancer treatment [J]. Int J Radiat Oncol Biol Phys, 2012, 83(4): 1169-1178.

10. Boylan CJ, Golby C, Rowbottom CG. A VMAT planning solution for prostate patients using a commercial treatment planning system[J]. Phys Med Biol, 2010, 55(14):395-404

11. Ming-Hsien Li, Sheng-Fang Huang, Chih-Chieh Chang, et al. Variations in dosimetric distribution and plan complexity with collimator angles in hypofractionated volumetric arc radiotherapy for treating prostate cancer[J]. Journal of Applied Clinical Medical Physics, 2018, 19(3 Suppl):93.

12. Rao M, Yang W, Chen F, et al. Comparison of Elekta VMAT with helical tomotherapy and fixed field IMRT: plan quality, delivery efficiency and accuracy[J]. Med Phys, 2010, 37(3):1350-1359.

13. Bedford JL. Treatment planning for volumetric modulated arc therapy[J]. Med Phys, 2009, 36(11): 5128-5138.

14. Mohler JL, Kantoff PW, Armstrong AJ, et al. Prostate cancer, version 2.2014[J]. J Natl Compr Canc Netw, 2014, 12(5): 686-718.

15. Mack Roach III, Gerald Hanks, Howard Thames Jr, et al. Defining biochemical failure following radiotherapy with or without hormonal therapy in men with clinically localized prostate cancer: Recommendations of the RTOG-ASTRO Phoenix Consensus Conference[J]. 65(4):965-974.

16. ICRU. Prescribing, recording and reporting photon-beam intensity-modulated radiation therapy(IMRT) [M]. ICRU Report 83. Oxford: Oxford University Press,2010,10(1):41-52

17. Yoo S, Wu QJ, Lee WR, et al. Radiotherapy treatment plans with RapidArc for prostate cancer involving seminal vesicles and lymph nodes[J]. Int J Radiat Oncol Biol Phys, 2010, 76(3): 935-942.

18. Melissa James, Gabrielle McLean, Stephen Williams, Bridget Robinson, \& Ma Yi. (2018). Complications of curative radiation treatment for early prostate cancer. Asia pacific Journal of Clinical Oncology, 14(18).

19. Chaswal V, Weldon M, Gupta N, et al. Commissioning and comprehensive evaluation of the ArcCHECK cylindrical diode array for VMAT pretreatment delivery QA[J]. J Appl Clin Med Phys, 2014, 15(4):4832-4832.

20. Dearnaley D, Syndikus I, Mossop H, et al. , non-inferiority, phase $3 \mathrm{CHHiP}$ trial[J]. Lancet Oncol, 2016, 17(8):1047-1060.

21. Guckenberger $M$, Richter $A$, Krieger $T$, et al. Is a single arc sufficient in volumetric-modulated arc therapy (VMAT) for complex-shaped target volumes?[J]. Radiother Oncol, 2009, 93(2): 259-265.

22. Gomez-Millan Barrachina J, Jerez Sainz I, Perez Rozos A, et al. Potential advantages of volumetric arc therapy in head and neck cancer[J]. Head Neck, 2015, 37(6): 909-914.

23. Chaswal V, Weldon M, Gupta N, et al. Commissioning and comprehensive evaluation of the ArcCHECK cylindrical diode array for VMAT pretreatment delivery QA[J]. J Appl Clin Med Phys, 2014, 15(4):4832-4832.

24. Ong A, Ang K, Master Z, et al. Intensity-Modulated Radiotherapy for Whole Pelvis Irradiation in Prostate Cancer: A Dosimetric and Plan Robustness Study between Photons and Protons[J]. 2018, 
6:11-19.

25. Gomez-Millan Barrachina J, Jerez Sainz I, Perez Rozos A, et al. Potential advantages of volumetric arc therapy in head and neck cancer[J]. Head Neck, 2015, 37(6): 909-914.

Figures
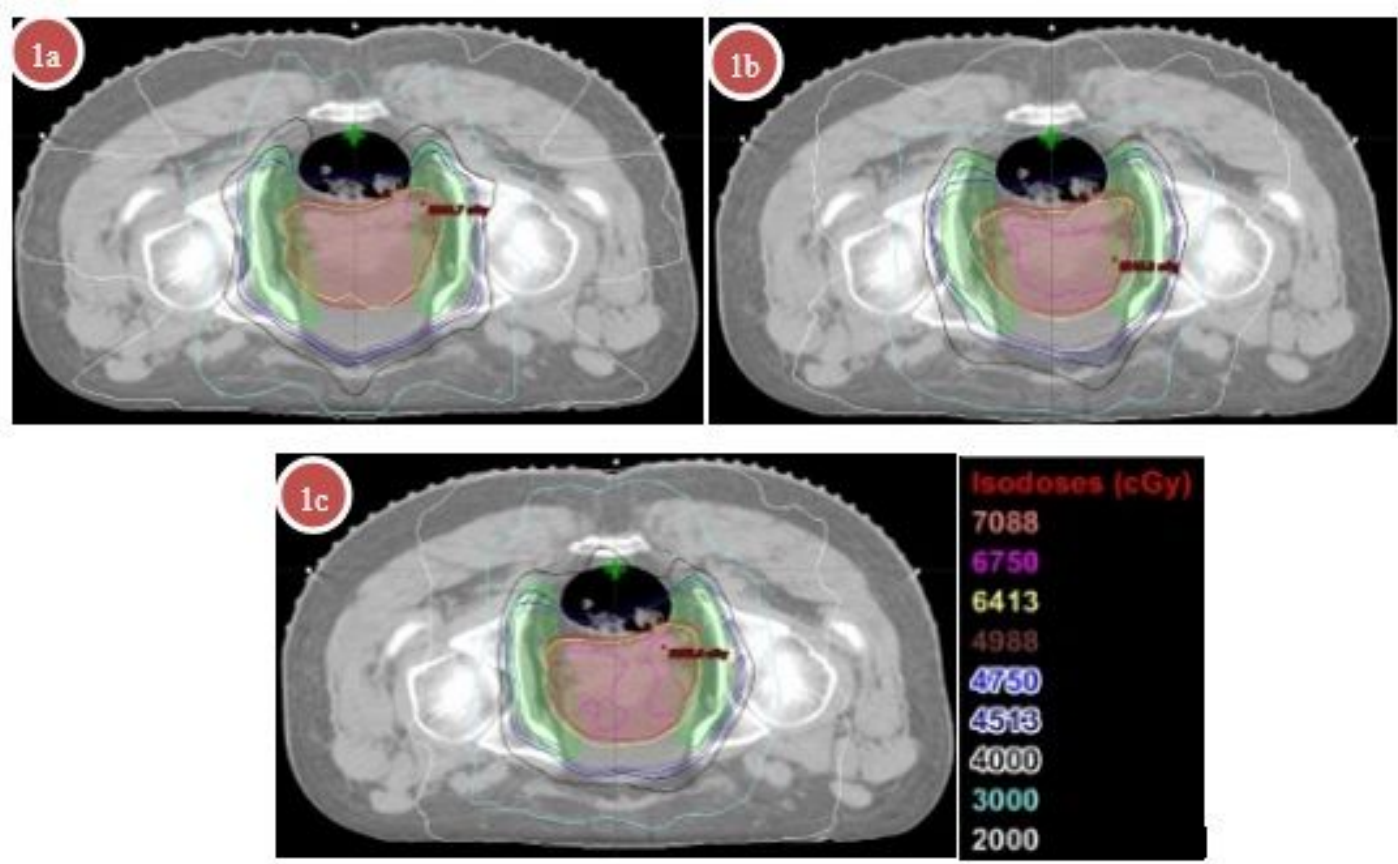

\section{Figure 1}

$1 \mathrm{a}$ is plan $9 \mathrm{~F}, 1 \mathrm{~b}$ is plan $1 \mathrm{ARC}$, and $1 \mathrm{c}$ is plan $2 \mathrm{ARC}$. The red line is PTV1, the green line is PTV2, and the other color lines are isodose lines. 